\title{
Various views on anonymity
}

To the editor - With regard to the November editorial on the subject of anonymous versus signed peer review, I believe that peer review would be vastly improved and be less damaging if reviews were signed. Furthermore the problem for journals is magnified by the major problem at funding agencies like the National Institutes of Health (NIH). There it is now possible to have your science judged by patently conflicted individuals, and your career destroyed.

\section{ALLEN Roses}

\section{Department of Neurology}

Duke University Medical Center

Durham, North Carolina 27710-2900, USA

To the editor - I notice that in your editorial you did not talk about the possibility of authors, not reviewers, being anonymous. I have yet to hear a compelling reason why reviewers should know who wrote the paper they are reviewing, and I can think of good reasons why they should not know. As the system stands now, reviewers are free to sign their name any time they wish, or remain anonymous if they wish, which is fine. Authors do not have the same option, but should.

\section{LARRY CAHILL \\ Center for the Neurology of Learning and \\ Memory, \\ University of California at Irvine, \\ Irvine, California 92717, USA}

To the editor - Senior scientists are precisely those who have the least to lose if their identities are known in the course of delivering an unfavorable opinion about an article. Many reviewers are at earlier stages in their careers and might be more reluctant to be candid. This is a strong reason for preserving anonymity. Nevertheless, there is a second problem that is inherent in the process. A reviewer, however senior or junior, may have an ax to grind, one either known or unknown to those submitting a paper. How can the editor control for this? One way is to make submissions anonymous, but we all know each other well enough that the reviewer often can guess who is submitting based on content or references. The second way is clearly more nebulous, and more troublesome to the

editor: vigilance for this possibility and even a mechanism for challenging the content of a review, unfavorable or not.

\section{RON LESSER}

Professor of Neurology and Neurosurgery

Johns Hopkins University

600 North Wolfe Street

Baltimore, Maryland 21287-7247, USA

To the editor - I would like to suggest a hybrid alternative to signed or anonymous reviews. This alternative involves having the two or three reviewers unmasked as to each others' identity but masking their identity from the authors. I have seen this work with the Journal of Clinical Endocrinology and Metabolism for more than two decades and like it because it puts a sense of peer pressure on the reviewers. The knowledge that an equally respected colleague will be able to judge both the depth and fairness of a review may result in more informative and sober reviews. I have no way of proving that this is so, except by asking people with experience what they think. I know, for example, that I have sometimes written to an editor privately commenting that the other reviewers' remarks were off base, unfair or, in some cases, a possible conflict of interest.

Julio V. SANTIAGo

Editor, Diabetes

Professor of Pediatrics and Medicine

Washington University School of Medicine

St. Louis, Missouri 63110, USA

To the editor - It is my observation that the quality of manuscript reviews has

\section{Live $E$. coli cells to treat uremia}

To the editor - In the August issue of Nature Medicine (2, 883-887; 1996), S. Prakash and T.M.S. Chang reported the administration of genetically engineered live $E$. coli cells to uremic rats in an attempt to reduce plasma urea levels. The encapsulated bacteria used the urea as a nitrogen source and, in so doing, lowered the plasma urea concentration to near normal levels. Although this could have exciting implications for the treatment of acute and chronic renal failure, decreased dramatically over the past five years. Reviewers with professional or economic conflicts of interest, or even mere personal animus, will say anything to block or to delay publication of important manuscripts. Editors seem helpless to deal with the controversies disingenuous reviews generate.

Deceptive techniques of manuscript reviewing may have metastasized from certain longstanding disreputable strategies used in grant peer reviews. Requiring signed reviews could be an effective way to speed the publication of important, scientifically sound research. Another practice that would eliminate the need for long contentious rebuttals by authors would be for editors to disqualify a review once it was shown to contain any demonstrably false statement. Disingenuous reviewers would not test the waters repeatedly if their efforts were thwarted by firm editorial responses.

If editors do not insist on signed reviews or are unwilling to reject misleading reviews out of hand, another deterrent might be the display of dishonest reviews and a brief rebuttal at a Web site, which would embarrass a journal's editors if not the anonymous reviewers. Restoring personal responsibility and integrity to manuscript and grant peer review will be necessary if there is to be a renaissance of creative science.

MiCHAEL SWIFT

The Institute for the Genetic Analysis of Common Diseases, Department of Pediatrics, New York Medical College, 4 Skyline Drive, Hawthorne, New York 10532, USA a number of aspects of the work are unclear, prompting several questions and concerns.

It is unclear why unilateral nephrectomy and contralateral ligation of the renal vessels was performed. This procedure leads to immediate renal failure and total anuria. Acidosis, azotemia, hyperkalemia and overhydration rise within hours, bringing the animals close to death in just a few days. Yet, Prakash and Chang report that their animals sur- 